\title{
Applications of vestibular-evoked myogenic potentials in vestibulogy. 1. Basic principles.
}

\author{
Mario Milkov
}

Department of Prosthetic Dental Medicine, Faculty of Dental Medicine, Medical University of Varna

\begin{abstract}
Diagnosis of vestibular disorders represents a serious challenge for modern otoneurology. In the recent decades, there is a gradual enrichment of the diagnostic tools enabling the objective assessment of the physiology of the vestibular system under normal and pathological conditions. Here belong the vestibular-evoked myogenic potentials (VEMPs). In the first of a series of review papers on this hot topic, the basic peculiarities of both types of the VEMPs, the cervical and ocular VEMPs, are summarized.
\end{abstract}

Key words: vestibular-evoked myogenic potentials, cervical vestibular-evoked myogenic potentials, ocular vestibular-evoked myogenic potentials, vestibular disorders, diagnosis
$\mathrm{V}$ estibular disorders are relatively common today and require timely and precise diagnosis. Loud clicks, short tone bursts, head taps and short duration transmastoid currents are all capable of activating vestibular receptors and evoking reflex changes in tonic electromyogram (EMG) activity within the sternocleidomastoid muscles (SCM) (3). Because they derive from averaged EMGs, the responses are termed 'vestibular evoked myogenic potentials' (VEMPs). The earliest response ipsilateral to a loud click, p13n23, is dependent upon vestibular activation, specifically saccular afferents.

In 1992, Colebatch and Halmagyi were the first to publish a report about the methods of cervical vestibular evoked myogenic potentials (2).

Vestibular-evoked myogenic potentials (VEMPs) are short latency manifestations of vestibulo-ocular and vestibulocollic reflexes that originate from the utricle and saccule. In the central nervous system, the cervical VEMPs (cVEMPs) are mediated by the vestibular nuclei and uncrossed medial vestibulospinal tract descending in the lower brainstem and spinal cord (24). The ocular VEMPs (oVEMPs) reflect the function of the vestibular nuclei and the crossed vestibulo-ocular reflex pathways in the medial longitudinal fasciculus. Involvement of the vestibular nuclei presents with abnormalities of both cVEMPs and oVEMPs. The medullary lesions of the descending medial longitudinal fasciculus or the spinal accessory nucleus impair cVEMPs while those of the medial longitudinal fasciculus, 
the crossed ventral tegmental tract, oculomotor nuclei and the interstitial nucleus of Cajal impair oVEMPs (24).

VEMP amplitudes are increased by threat and fear (23) showing that there are also other inputs to consider. The cVEMPs are not simple saccule reflexes, but have multiple inputs.

The output for cVEMP responses is by definition the SCM innervated by the accessory $11^{\text {th }}$ cranial nerve. If either one of these structures were disturbed, one would expect alterations in the cVEMP as well (38). There are many other muscles that activate the neck and are relevant to posture that are activated by sound, too. Additionally, when the semicircular canals are sensitive to sound, it is very likely that vertical eye muscles on both sides of the head are activated by the VEMP protocol. Thus the ipsilateral wiring assumptions may be incorrect in certain ear diseases.

VEMPs are recorded using an evoked response computer, a sound generator, and surface electrodes to pick up neck muscle activation or other muscles if this is of interest. A Bio-Logic Navigator Pro for VEMP recordings does nearly all of the work, and sends the results to a desktop computer (38).

VEMP testing is not hard, but there are a lot of technical pitfalls. The basics can be learned by a technician in about 30 minutes. It is a very big response, and as long as the person doing the test is attentive to details (getting the sound in both ears with proper placement of inserts or headphones, having the person lift his/her head through the entire trial, electrodes in the right place with proper impedance), it is very straightforward. The details take much longer to learn (38).

The cVEMP response consists of an initial positivity ( $\mathrm{p} 1$ or $\mathrm{p} 13)$ followed by a negativity ( $\mathrm{n} 1$ or $\mathrm{n} 23$ ). It is an evoked potential. Although P1 is positive, it is shown negative on many cVEMPs, because of electrode placement (basically putting them on backwards). The amplitude is the most reliable measure of the cVEMP response (17).

Later cVEMP components have a lower stimulus threshold and are thought to be nonvestibular. As VEMPs are easily elicited without the need for EMG rectification, and EMG spikes occur at roughly the same latency as the waves in a cVEMP, coherent spike trains are a reasonable alternative explanation. In other words, later waves may all be part of the same response (38). A VEMP system that rectifies is not necessary (10). Rectification can correct in part for technical errors involving head positioning, at the price of adding more 'noise' to the entire system. Higher than normal thresholds or low amplitudes may be found in persons with saccule disorders as well as conductive hearing loss. Reduced amplitudes are commonly found in vestibular nerve disturbances.

Because the response is generally ipsilateral, theoretically, bilateral stimuli and bilateral recording can be used to reduce the number of trials (41). The cVEMP is generally quick and easy to obtain because it is a strong potential and only requires about a minute of stimulation to get 100 presentations.

As the entire vestibular nerve is stimulated by galvanic input, galvanic VEMPs would be insensitive to partial nerve lesions (i.e. failed vestibular nerve section), but also quite sensitive to complete vestibular nerve loss. Thus an absent galvanic VEMP might be used as a rationale to avoid doing more surgery (38). For the same reason, galvanic VEMPs should also not be able to differentiate between endorgan (saccule) damage and inferior vestibular nerve damage because one would expect that the galvanic VEMP would be present even if the inferior vestibular nerve were damaged.

With supra-threshold acoustic stimuli (e.g. 100 $\mathrm{dB}$ nHL), optimally at $500 \mathrm{~Hz}$, reflective nerve impulses of mainly irregular otolith neurons may be activated (Curthoys IS). The generated electrical potentials can be objectified by means of surface electromyography of cervical (sternocleidomastoid muscle) and ocular measurements (oblique and inferior rectus muscles) resulting in the typical VEMP curves $(2,29)$. Their morphology (amplitude and latency) in the time course and side comparison (amplitude ratio, latency ratio) is used as measure for the function. Regarding the origin of the ocular VEMP measurements, controversial discussions were conducted during the last years, especially with regard to the double innervation of the sacculus (40). Nowadays it is accepted that cVEMP and oVEMP in air conduction are an indicator for the preponderant sacculus function (sacculo-collic reflex) or utriculus function (utriculo-ocular reflex) $(8,10)$. Both methods are widely applied in daily clinical practice as well as in the context of otorhinolaryngological specific expert opinion. VEMP can be applied in all ages (36).

Prior to each VEMP examination, ear microscopy (if needed removal of earwax, assessment of the 
condition of the auditory meatus and eardrum) as well as audiological diagnostics (e.g. tympanometry, tone audiometry, measurement of air and bone conduction thresholds) are recommended. According to its severity, conductive hearing loss leads to a reduction of all VEMP responses. Sensory hearing loss such as deafness does not impair the examination of the VEMP (38).

For daily routine, measurements by means of air conduction are currently best evaluated. Bone conduction stimulation is possible in cases of conductive hearing loss. For examination by air conduction, in-ear headphones or earphones are suitable. A VEMP module is precondition for VEMP measurement. The surface electrodes can be placed for example in the area of the upper third of both sternocleidomastoid muscles, in the middle of the forehead (neutral electrode), and in the area of the jugulum (reference electrode). For a rapid and undisturbed measurement, low impedances (e.g. < $5 \mathrm{k} \Omega$ ) are necessary. For measurement in sitting or lying position, the head is turned to the contralateral side and slightly inclined or lifted so that the sternocleidomastoid muscle of the stimulated side is contracted. According to the current international recommendations (25), first the frequency of the best acoustic sensitivity of the otolith organs is selected as stimulus frequency $(500 \mathrm{~Hz})$. At this frequency, the lowest thresholds and the highest amplitudes are found. As stimulus, click or burst stimuli are suitable. Clicks possess relatively broad frequency response with high-frequency parts and the frequency-specific stimulation is more unspecific than for burst stimuli (37). For stimulation, for example 50-100 repetitions are suitable until a typical amplitude complex at e.g. $100 \mathrm{~dB}$ results.

The cVEMP are inhibitory reflex responses. During ipsilateral stimulation, as objective sign of an intact sacculo-collic reflex, ipsilateral biphasic muscle potentials are found (positive potential at about 13 $\mathrm{ms}$ and negative potential at about $23 \mathrm{~ms}$ ) as well as acoustically generated potentials at about $33 \mathrm{~ms}$ and about $44 \mathrm{~ms}$ of which the properties are not exactly evaluated yet (29). The amplitudes (peak-to-peak measurement) are subject to fluctuations according to the patient's age and amount to about $<500 \mu \mathrm{V}$. Since the nerve fibers of the utriculo-ocular reflex cross centrally to the contralateral side, the measurement of the muscle potentials for oVEMP is performed in air or bone conduction of the contralateral side. The surface electrodes for analysis of the oVEMP can be placed for example bilaterally at the lower edge of the orbita and the reference electrode parallel below. For the neutral electrode, the middle of the forehead is recommended. Recently, a modified electrode position that also promises high oVEMP amplitudes was suggested (9). During stimulation, the patient has to look upwards in order to contract the outer eye muscles (oblique and inferior rectus muscles).

The oVEMP are excitatory electromyographic responses. An electromyographic control of the muscle tension during the measurement is not necessary. The classical oVEMP potential is also biphasic (negative potential at about $10 \mathrm{~ms}$, positive potential at about $5 \mathrm{~ms}$ ). The amplitudes are lower than for cVEMP (about $<20 \mu \mathrm{V}$ ) and achieve partly the nanovolt range $(28,33,34)$. The angle in upward direction and the horizontal gaze deflection as well as the body position influence the oVEMP results. The head rotation and the vision do not exert any significant impact on the results (7).

The VEMP measurements in air conduction (500 $\mathrm{Hz}$ ) are the classical stimulation method in daily practice. For assessment of the results, the amplitude (measurement between the amplitude maxima $[\mu \mathrm{V}])$ of the received measurement and the respective latency (s) as well as the amplitude ratio (asymmetry ratio, AR, in \%). For AR calculation, the higher (h) and lower (l) amplitudes (A) of both sides are considered: $\mathrm{AR}=100(\mathrm{Ah}-\mathrm{Al}) /(\mathrm{Ah}-\mathrm{Al})$. An amplitude ratio of $>50 \%$ is pathological (25). Thus finding quantification is possible. The data of the reference ranges varies relevantly in the literature available $(25,29)$.

Pathological latencies and amplitude ratios indicate an impairment of the respective reflex. The entire reflex chain has to be considered because the otolith organs as well as the superior and inferior vestibular nerves, central pathways, or, rarely, even muscle functions may be impaired (24). The VEMP application requires a previous differential diagnostic classification of the complaints.

Different stimulation modalities (e.g. type of stimulus, location of stimulation, stimulus frequency) have a significant impact on the VEMP amplitudes $(28,29,33,34)$.

Both cVEMP and oVEMP in air conduction (500 $\mathrm{Hz})$ reflect the main sacculus and utriculus functions. Regarding the cVEMP, the muscle pre-tension must be taken into account for evaluation of the reference range. 
Besides stimulation by air conduction, the stimulation via bone conduction is described in the literature available, e.g. by means of conventional bone conduction headphones, mini-shakers, or reflex hammers.

Stimulation via bone conduction may be performed in the middle of the forehead or in the area of the mastoid $(29,33,34)$. It may be suitable when there is conductive impairment (e.g. eardrum perforation or open mastoid cavity). There are different mechanisms for stimulation of the otolith organs (10). Bone conduction stimuli should rather lead to stimulation of the otolith membrane while air conduction stimuli should lead to direct stimulation of acoustically sensitive vestibular hair cells (10).

Besides the optimal stimulus frequency of $500 \mathrm{~Hz}$, the acoustic stimulation of the otolith organs is also possible with other stimulus frequencies $(<100$ $\mathrm{Hz}->4 \mathrm{kHz}$ ).

Since VEMP examinations are supra-threshold diagnostic measures, it is recommended to apply the VEMP stimulus in a way that the acoustic energy is kept low.

The application of chirp stimuli seems to be a promising modification of VEMP analysis, especially in the context of diagnostics of frequency dynamic changes of the otolith organs (37). Chirps are special stimuli with a frequency range that can be flexibly designed. High VEMP amplitudes can be generated with chirp stimuli constructed for narrowband and broadband frequency structures (37). Recently, numerous articles show that VEMP measurements contribute to a decisive improvement of vestibular diagnostics (38). For the first time, it is possible to objectively assess the involvement of the otolith organs in vestibular syndromes (22].

In the context of vestibular neuritis, cVEMP and oVEMP allow statements about an involvement of the otolith organs. If only the cVEMP measurements are pathological, an involvement of the inferior vestibular nerve (or the sacculus function) can be assumed. If additionally oVEMP examination is conspicuous, an involvement of the superior vestibular nerve (or the utriculus function) can be expected (38).

The VEMPs play a key role in the diagnostics of the semicircular canal dehiscence. There is conspicuous cVEMP in $85 \%$ of the cases. In $62 \%$ of the patients, oVEMP are pathological. A dehiscence syndrome is suspected when reduced VEMP thresholds are found in air conduction stimulation with $500 \mathrm{~Hz}$ as well as increased VEMP amplitudes (11). Both cVEMP and oVEMP amplitudes and cVEMP thresholds correlate with the severity of a dehiscence (16). In a multicentre study, both cVEMP thresholds and oVEMP amplitudes are good diagnostic tests for identifying the superior semicircular canal dehiscence (15). The sensitivity and specificity of these individual tests may vary slightly depending on testing parameters used.

Pathological oVEMP are established in the context of benign positional vertigo (18). The correlation of this disease with a disturbed utriculus function can be easily objectified (18) which should be confirmed formerly by complex rotatory tests.

The VEMP examinations are suitable for all peripheral vestibulopathies $(29,33,34)$ in order to identify otolith involvement. These results provide detailed information about the otolith function and thus differential diagnostic information in the context of peripheral vestibulopathies. They may facilitate therapy decisions.

While bone conducted stimuli at most sites work through displacement of the otolith membrane, bone conducted oVEMPs may work through a direct action on utricular hair cells (12). The VEMP routinely uses stimulus frequencies of 500 and 1000 $\mathrm{Hz}$, and otoliths are established incontrovertibly by clinical and neural evidence as the stimulus source (13). These stimuli can cause noise-induced hearing loss due to high sound exposure levels. With noise exposure calculation tool and proper safety sample protocols in place, clinicians may reduce or eliminate this risk to their patients (28).

When completing VEMP testing, the stimulus is by approximately $3 \mathrm{~dB}$ higher in a child's ear; a 500 $\mathrm{Hz}$ tonebursts is recommended over a click as it has lower peak-to-peak equivalent sound pressure levels compared to the click, and both duration and intensity should be considered when choosing VEMP stimuli in children (31).

The VEMPs can be elicited with electrical stimulation in a proportion of children with cochlear implants thus demonstrating current spread from the cochlea to the vestibular system (27). Electric VEMP presence in acoustically nonresponsive ears, along with the shorter latencies of electrically driven VEMPs, suggests that electrical current can bypass the otoliths and directly stimulate vestibular neural elements (27).

This diagnostic methods using VEMPs such as cVEMP and/or oVEMP have already been applied 
in the assessment of central and peripheral otolithic function (24), benign paroxysmal positional vertigo (22), idiopathic otolithic vertigo (22), Ménière's disease (22), vestibular neuritis $(1,10,22,35)$, central vertigo/dizziness (22), vertigo, dizziness and imbalance (30), vestibular nerve tumours such as acoustic neuromas (3), superior canal dehiscence $(32,39)$, vestibular migraine $(22,24)$, otosclerosis, multiple sclerosis $(5,6,14,24)$, unilateral cerebellar infarctions (24), etc. A completing VEMP analysis is suitable in the context of traumatic disorders and peripheral vestibulopathies with changed inner ear mechanics (37). Frequency dynamic changes are described for dehiscence syndrome, too (20). Recently, hearing loss during VEMP diagnostics has been reported, too (21).

Further interdisciplinary research is needed to further clarify the unsolved issues of the diagnostic role of the VEMPs.

\section{References}

1. Adamec I, Skorić MK, Handžić J, Barušić AK, Bach I, Gabelić T, et al. The role of cervical and ocular vestibular-evoked myogenic potentials in the follow-up of vestibular neuritis. Clin EEG Neurosci. 2014;45(2):129-136.

2. Colebatch JG, Halmagyi GM. Vestibular evoked potentials in human neck muscles before and after unilateral vestibular deafferentation. Neurology. 1992;42(8):1635-1636.

3. Colebatch JG. Vestibular evoked potentials. Curr Opin Neurol. 2001;14(1):21-26.

4. Colebatch JG, Rosengren SM. Safe levels of acoustic stimulation: comment on 'Effects of acoustic stimuli used for vestibular evoked myogenic potential studies on the cochlear function'. Otol Neurotol. 2014;35(5):932-933.

5. Crnošija L, Krbot Skorić M, Gabelić T, Adamec I, Habek M. Vestibular evoked myogenic potentials and MRI in early multiple sclerosis: Validation of the VEMP score. J Neurol Sci. 2017;372:28-32.

6. Gabelić T, Krbot Skorić M, Adamec I, Barun B, Zadro I, Habek M. The vestibular evoked myogenic potentials (VEMP) score: a promising tool for evaluation of brainstem involvement in multiple sclerosis. Eur J Neurol. 2015;22(2):261-269.

7. Govender S, Rosengren SM, Colebatch JG. The effect of gaze direction on the ocular vestibular evoked myogenic potential produced by airconducted sound. Clin Neurophysiol. 2009;120(7):1386-1391.

8. Govender S, Dennis DL, Colebatch JG. Vestibular evoked myogenic potentials (VEMPs) evoked by air- and bone-conducted stimuli in vestibular neuritis. Clin Neurophysiol. 2015;126(10):2004-2013.

9. Govender S, Cheng PY, Dennis DL, Colebatch JG. Electrode montage and gaze effects on ocular vestibular evoked myogenic potentials (oVEMPs). Clin Neurophysiol. 2016;127(8):2846-2854.

10. Govender S, Dennis DL, Colebatch JG. Frequency and phase effects on cervical vestibular evoked myogenic potentials (cVEMPs) to airconducted sound. Exp Brain Res. 2016;234(9):2567-2574.

11. Govender S, Fernando T, Dennis DL, Welgampola MS, Colebatch JG. Properties of 500Hz air- and bone-conducted vestibular evoked myogenic potentials (VEMPs) in superior canal dehiscence. Clin Neurophysiol. 2016;127(6):2522-2531.

12. Govender S, Colebatch JG. Location and phase effects for ocular and cervical vestibular-evoked myogenic potentials (VEMPs) evoked by bone-conducted stimuli to midline skull sites. J Neurophysiol. 2017 Dec 13. doi: 10.1152/jn.00695.2017.

13. Grant W, Curthoys I. Otoliths - accelerometer and seismometer; implications in vestibular evoked myogenic potential (VEMP). Hear Res. 2017;353:26-35.

14. Güven H, Bayır O, Aytaç E, Ozdek A, Comoğlu SS, Korkmaz H. Vestibular-evoked myogenic potentials, clinical evaluation, and imaging findings in multiple sclerosis. Neurol Sci. 2014;35(2):221-226.

15. Hunter JB, O'Connell BP, Wang J, Chakravorti S, Makowiec K, Carlson ML, et al. Correlation of superior canal dehiscence surface area with vestibular evoked myogenic potentials, audiometric thresholds, and dizziness Handicap. Otol Neurotol. 2016;37(8):1104-1110.

16. Hunter JB, Patel NS, O’Connell BP, Carlson ML, Shepard NT, McCaslin DL, et al. Cervical and ocular VEMP testing in diagnosing superior semicircular canal dehiscence. Otolaryngol Head Neck Surg. 2017;156(5):917-923.

17. Isaradisaikul S, Navacharoen N, Hanprasertpong C, Kangsanarak J. Cervical vestibular-evoked myogenic potentials: norms and protocols. Int J Otolaryngol. 2012;2012:913515. doi: 10.1155/2012/913515.

18. Kim EJ, Oh SY, Kim JS, Yang TH, Yang SY. Persistent otolith dysfunction even after successful repositioning in benign paroxysmal positional vertigo. J Neurol Sci. 2015;358(1-2):287-293.

19. Lee KJ, Kim MS, Son EJ, Lim HJ, Bang JH, Kang JG. The usefulness of rectified VEMP. Clin Exp Otorhinolaryngol. 2008;1(3):143-147.

20. Manzari L, Burgess AM, McGarvie LA, Curthoys IS. An indicator of probable semicircular canal dehiscence: ocular vestibular evoked myogenic potentials to high frequencies. Otolaryngol Head Neck Surg. 2013;149(1):142-145.

21. Mattingly JK, Portnuff CD, Hondorp BM, Cass SP. Sudden bilateral hearing loss after cervical and ocular vestibular evoked myogenic potentials. Otol Neurotol. 2015;36(6):961-964.

22. Murofushi T. Clinical application of vestibular evoked myogenic potential (VEMP). Auris Nasus Larynx. 2016;43(4):367-376.

23. Naranjo EN, Cleworth TW, Allum JH, Inglis JT, Lea J, Westerberg BD, et al. Vestibulo-spinal and vestibulo-ocular reflexes are modulated when standing with increased postural threat. J Neurophysiol. 2016 Feb 1;115(2):833-42.

24. Oh SY, Kim HJ, Kim JS. Vestibular-evoked myogenic potentials in central vestibular disorders. J Neurol. 2016;263(2):210-220.

25. Papathanasiou ES, Murofushi T, Akin FW, Colebatch JG. International guidelines for the clinical application of cervical vestibular evoked myogenic potentials: an expert consensus report. Clin Neurophysiol. 2014;125(4):658-666.

26. Parkes WJ, Gnanasegaram JJ, Cushing SL, McKnight CL, Papsin BC, Gordon KA. Vestibular evoked myogenic potential testing as an objective measure of vestibular stimulation with cochlear implants. Laryngoscope. 2017;127(2):E75-E81. 
27. Portnuff CDF, Kleindienst S, Bogle JM. Safe use of acoustic vestibular-evoked myogenic potential stimuli: protocol and patient-specific considerations. J Am Acad Audiol. 2017;28(8):708-717.

28. Rosengren SM, Govender S, Colebatch JG. The relative effectiveness of different stimulus waveforms in evoking VEMPs: significance of stimulus energy and frequency. J Vestib Res. 2009;19(1-2):33-40.

29. Rosengren SM, Welgampola MS, Colebatch JG. Vestibular evoked myogenic potentials: past, present and future. Clin Neurophysiol. 2010;121(5):636-651

30. Skorić MK, Adamec I, Pavičić T, Pavlović I, Ruška B, Crnošija L, et al. Vestibular evoked myogenic potentials and video head impulse test in patients with vertigo, dizziness and imbalance. J Clin Neurosci. 2017;39:216-220.

31. Thomas MLA, Fitzpatrick D, McCreery R, Janky KL. Big stimulus, little ears: safety in administering vestibular-evoked myogenic potentials in children. J Am Acad Audiol. 2017;28(5):395-403.

32. Verrecchia L, Westin M, Duan M, Brantberg K. Ocular vestibular evoked myogenic potentials to vertex low frequency vibration as a diagnostic test for superior canal dehiscence. Clin Neurophysiol. 2016;127(4):2134-2139.

33. Walther LE, Hörmann K, Pfaar O. Die Ableitung zervikaler und okulärer vestibulär evozierter myogener Potenziale: Teil 1: Anatomie, Physiologie, Methodik und Normalbefunde. HNO. 2010;58(10):1031-1045.

34. Walther LE, Hörmann K, Pfaar O. Die Ableitung zervikaler und okulärer vestibulär evozierter myogener Potenziale. Teil 2: Einflussfaktoren, Bewertung der Befunde und klinische Bedeutung. HNO. 2010a;58(11):1129-1142.

35. Walther LE, Blödow A. Ocular vestibular evoked myogenic potential to air conducted sound stimulation and video head impulse test in acute vestibular neuritis. Otol Neurotol. 2013;34(6):1084-1089.

36. Walther LE, Brusis T. Aus der Gutachtenpraxis: Zeitgemäße, objektive Begutachtung der peripheren vestibulären Rezeptorfunktion (5-Rezeptoren-Diagnostik).Laryngorhinootologie. 2013;92(3):189-192.

37. Walther LE, Cebulla M. Tipps \& Tricks - Vestibulär evozierte myogene Potenziale: Multifrequenzanalyse der Otolithenfunktion mittels ChirpStimulation.Laryngorhinootologie. 2016;95(8):530-533.

38. Walther LE. Current diagnostic procedures for diagnosing vertigo and dizziness. GMS Curr Top Otorhinolaryngol Head Neck Surg. 2017;16: Doc02. doi:10.3205/cto000141.

39. Welgampola MS, Myrie OA, Minor LB, Carey JP. Vestibular-evoked myogenic potential thresholds normalize on plugging superior canal dehiscence. Neurology. 2008;70(6):464-772.

40. Welgampola MS, Carey JP. Waiting for the evidence: VEMP testing and the ability to differentiate utricular versus saccular function. Otolaryngol Head Neck Surg. 2010;143(2):281-283.

41. Young YH. Vestibular evoked myogenic potentials: optimal stimulation and clinical application. J Biomed Sci. 2006;13(6):745-751. 